\title{
The Difference Between the Perception of Absolute and Relative Motion: a Reaction
} Time Study

\author{
JEROEN B. J. SMEETS,* ELI BRENNER* \\ Received 25 March 1993; in revised form 27 May 1993
}

\begin{abstract}
We used a reaction-time paradian to examize the extent to which motion detection depends on relative motion. In the absence of relative notion, the responses could be described by a simple model based on the detection of a fixed charee in position. If relative motion was present, the responses could be modelled using characteribtics of motion detectors. Comparing reaction times when relative and absolute velocity are equal with ones when relative velocity is twice the absolute velocity reveals that these detectors measure relative motion.
\end{abstract}

Human Eye movements Motion perception Frame of reference Velocity

\section{INTRODUCTION}

In the study of motion perception, many authors use a fixation point to suppress eye movements. Apart from defining the orientation of the eye, and thus the retinal slip caused by the stimulus, this fixation point also serves as a visual reference. Thus, the use of a fixation point makes it irrelevant whether motion is perceived relative to the retina, to the head, or to an external frame of reference (the fixation point).

Brenner (1991) showed that when motion relative to our head is in conflict with that relative to a visual reference, we rely on motion relative to the visual reference. We recently showed (Brenner \& Smeets, 1993) that position (relative to ourselves) and velocity (relative to a visual reference) are perceived independently. Without relative motion, we probably must rely on our perception of changes in position (using non-retinal information) to detect that an object has moved. The distinction between motion and change of position has been discussed for over a century (Exner, 1888).

An interesting example of the importance of the distinction between (relative) motion and change of (absolute) position can be found in two models proposed to explain why reaction time to movement onset depends on stimulus velocity. Based on experiments on the onset of optokinetic nystagmus in rabbits, Collewijn (1972) proposed a model based on the detection of a fixed change of position. On the other hand, van den Berg and van de Grind (1989) described human reaction times [measured by Tynan and Sekuler (1982)] using a model

\footnotetext{
*Vakgroep Fysiologie, Erasmus Universiteit Rotterdam, Postbus 1738 ,
} NL-3000 DR Rotterdam, The Netherlands. based on characteristics of bilocal motion detectors. Each model fits the data the authors tried to explain, and performs worse on the other set.

Apart from the different species and motor systems studied, an important difference between the two experiments is that the rabbits in Collewijn's (1972) experiment had no visual reference, whereas the human subjects in Tynan and Sekuler's experiment had a fixation point. We hypothesize that the difference between absolute and relative motion is responsible for the outcomes leading to the different models.

To test this hypothesis, we determined reaction times to motion onset for various velocities of the target. We compared reaction times for stimuli with only absolute motion of the target to those for stimuli with target motion relative to a background, which was either static or moving.

\section{METHODS}

\section{Experimental set-up}

Nine colleagues, who were aware of the purpose of the experiment, served as subjects. They sat in front of a large screen with their arm resting on a table. The stimulus [see Fig. 1(a)] consisted of a red background (a high contrast random pixel array, $8 \times 8^{\circ}$ ) and a green target (a $0.1^{\circ}$ radius disk). Both the background and the target were projected via servo-controlled mirrors onto the screen. The mirrors allowed us to move both patterns independently in the horizontal direction. The red pattern was covered in the area through which the green dot could move, so that no change of contours was visible. Motion of the arm was recorded by means of an infra-red marker taped to the wrist and 
(a)

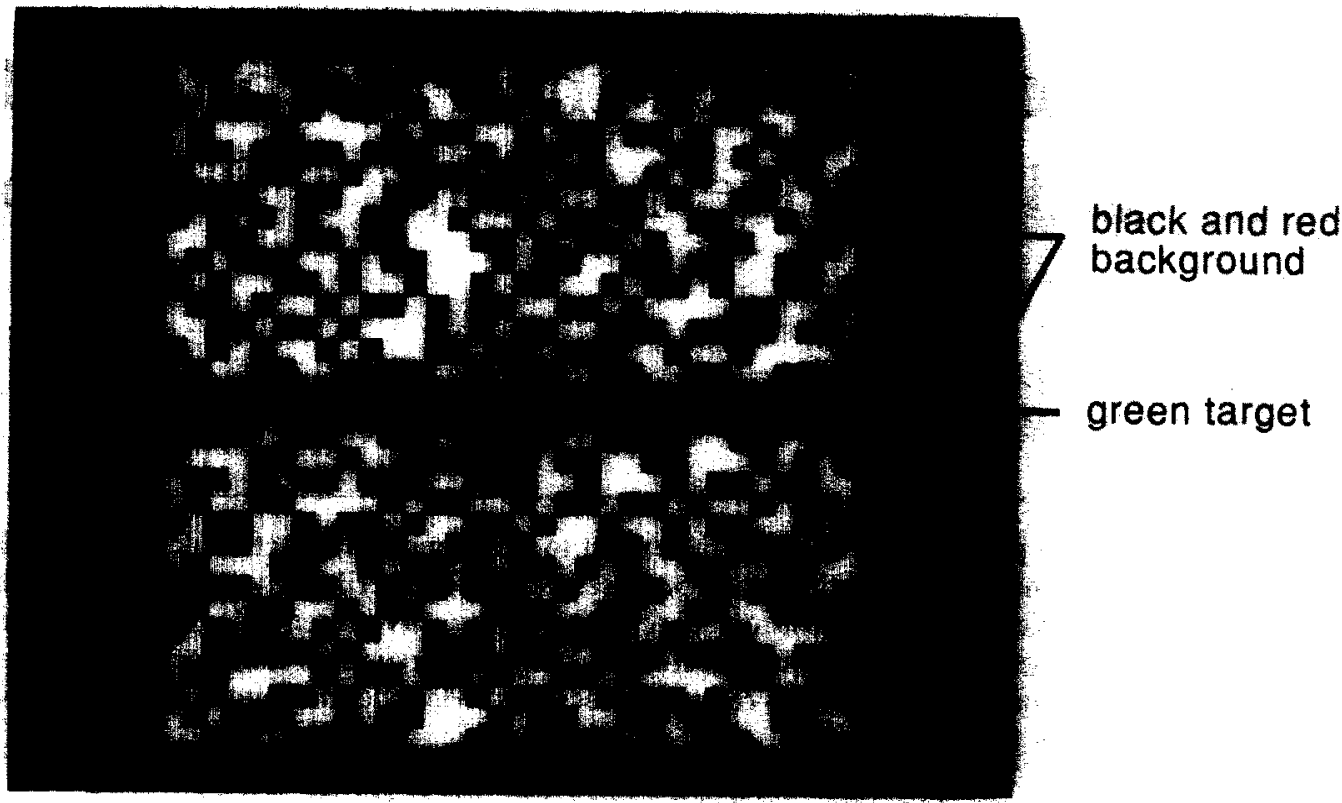

(b)

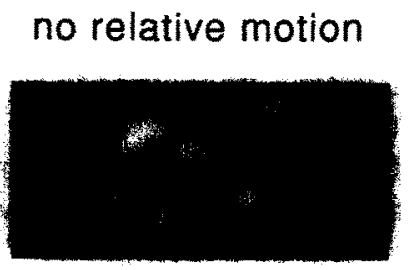

normal relative motion
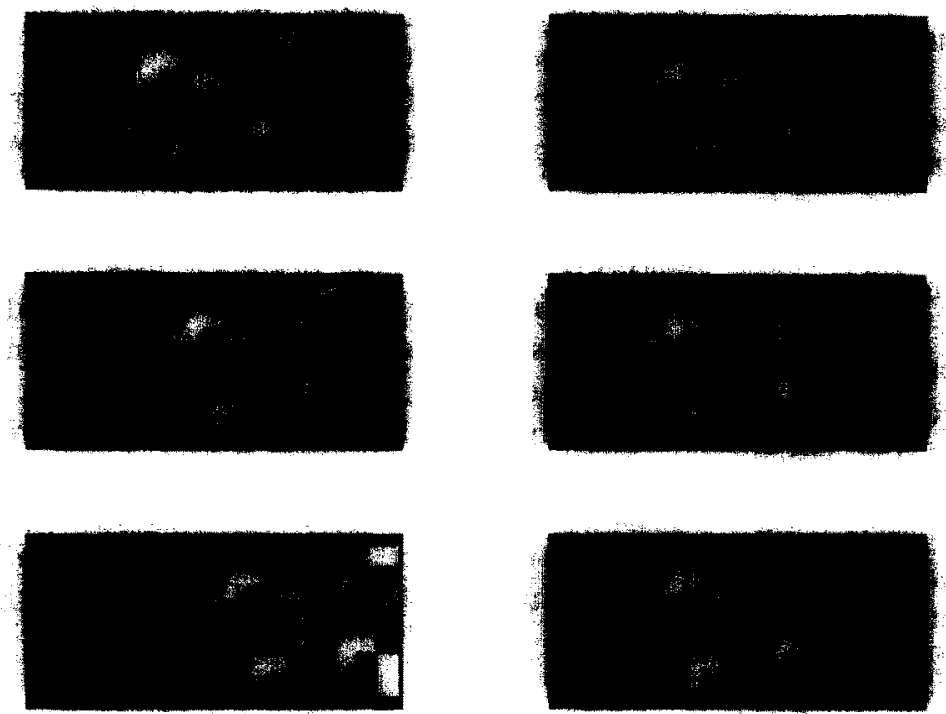

double relative motion
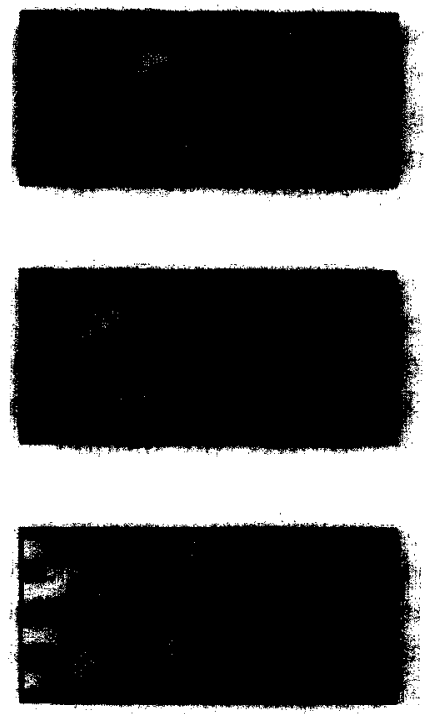

FIGURE 1. (a) Schematic drawing of the stimulus configuration used in the experiment. The stimuli had a luminance of about $40 \mathrm{~cd} / \mathrm{m}^{2}$ and were presented in a completely dark room. (b) Scheme of the motion of target and background in the three relative motion conditions.

an Optotrak motion analysis system (Northern Digital Inc.). The same system also recorded the movement of the mirrors.

Subjects were instructed to lift their arm as soon as they saw the green spot move. They knew that there were three possible stimuli [see Fig. 1(b)]: the background could remain static (normal relative motion), or move with the same speed as the target, either in the same direction as the target (no relative motion) or in the direction opposite to the target (double relative motion).
A warning tone was given $500-1000 \mathrm{msec}$ before the target started to move.

For each trial, data were collected at $500 \mathrm{~Hz}$ for $1500 \mathrm{msec}$, starting $300 \mathrm{msec}$ before the target motion onset. Seven target velocities were used $(0.28-17.8 \mathrm{deg} / \mathrm{sec})$, each with the three above mentioned relative motion conditions. The 21 possible stimuli were presented in random order. A pilot experiment showed larger variability in reaction time for low target velocities. We therefore used a different number of trials 
for each target velocity, increasing with one trial with each velocity step from three trials for the fastest to nine trials for the slowest target. This resulted in similar standard errors for all target velocities.

\section{Data analysis}

In a previous study (Brenner \& Smeets, 1993), we found that the velocity of the hand hitting a moving target depends on the velocity of the target. As the response-delay of a device with a fixed threshold (like a switch) depends on the speed at which the hand starts to move (see e.g. Anson, 1989), a more precise method was used to determine the reaction time. The onset of motion of both the arm and the mirrors were determined from the position data after low-pass filtering with a fourthorder Butterworth filter (Ackroyd, 1973). The filter was applied in both forward and backward direction to prevent phase shift, with an effective cut-off frequency of $100 \mathrm{~Hz}$. The latency of motion onset was defined as the last sample on which the velocity differed by less than one standard deviation from the value during the first $300 \mathrm{msec}$. The reaction time was defined as the difference between the measured onset of motion of the hand and that of the mirror. To eliminate responses not correlated to the visual stimulus, trials with a reaction time $<125 \mathrm{msec}$, or a reaction time more than three times the average reaction time for that condition were excluded from further analysis. If the subject did not respond within the $1200 \mathrm{msec}$ during which data were collected a reaction time of $1200 \mathrm{msec}$ was scored. This occurred six times, all at the lowest stimulus velocities. For all stimulus conditions, at least $90 \%$ of the trials could pass these criteria.

Two models were used to described the experimental data. The first one (position model) was proposed by Collewijn (1972). According to him, the reaction time $(R T)$ is the sum of a stimulus independent time $R T_{0}$ (required for neural processing and transportation) and the time it takes the stimulus (with an angular velocity $v$ ) to cover a certain angular distance $d$ on the retina:

$$
R T=R T_{0}+d / v .
$$

The second model (velocity model) was proposed by van den Berg and van de Grind (1989). They also assume a stimulus independent part $\left(R T_{0}\right)$, but instead of the duration of the displacement over a fixed distance $d$, they use the delay $\tau$ of a bilocal motion detector. This delay depends on the detector's tuning velocity $v: \tau=\tau_{0} /\left(v_{0} / v\right)$ with $\tau_{0}$ and $v_{0}$ heing the delay and tuning velocity of a certain detector. If we substitute the constant $c$ for $\tau_{0} / v_{0}$, this yields for the reaction time:

$$
R T=R T_{0}+c / \sqrt{ } v
$$

The experimentally testable difference between the two models is the linear vs square-root dependence on the reciprocal velocity. After calculating the mean reaction time and its standard error (averaged over all subjects) for each stimulus condition, we fitted both the position model and the velocity model to the data [Levenberg-Marquardt method (Press, Flannery, Teukolsky \& Vetterling, 1987)]. We did so separately for each relative motion condition. Two parameters were adjusted by the fitting procedure: $R T_{0}$ and $c$ or $d$.

\section{RESULTS}

In Fig. 2 we plot the reaction time, averaged over all trials of all subjects, as a function of the target velocity. The three symbols portray the different motion relative to the background. It is directly clear that motion of the background changes the reaction time: motion of the background in the same direction as the target (no relative motion) increases the reaction times to low stimulus velocities dramatically, whereas motion of the background in the direction opposite to the target (double relative motion) decreases these reaction times. The reaction times for targets in the double relative motion condition are similar to those for targets with double the velocity in the normal relative motion condition, so the velocity relative to the background seems to determine $R T$ (see Fig. 3). The results of the fits of both models for each condition are shown in Table 1.

For two conditions, the data differ significantly from the best fit of one of the models: the position model cannot fit the trials with double relative motion, and the velocity model cannot fit the trials without relative motion. For the trials with normal relative motion, the velocity model performs better than the position model.

The fits of the velocity model to the two conditions with relative motion yield approximately the same values for $R T_{0}$, whereas the values for $c$ clearly differ. These values are obtained assuming that $v$ in equation (2) indicates the (retinal) target velocity. If we assume that the velocity detectors measure relative motion instead of absolute (retinal) motion, we can fit equation (2) again to the data with relative motion. This fit yields $R T_{0}=197$ and $c=80$, with $\chi^{2}=4.9$. In Fig. 3, the data of the conditions with relative motion are plotted as a function of the velocity of the target relative to the background.

Our suggestion that motion detectors determine the $R T$ for the conditions with normal and double relative motion, whereas detection of a change in position does so when there is no relative motion, yields a prediction for $R T_{0}$. Assuming that its value is independent of the stimulus, $R T_{0}$ should be equal for all conditions. The obtained values for $R T_{0}$ are indeed approximately equal for the best fits (the position model for the condition without relative motion and the velocity model for the two conditions with relative motion).

The results of the experiment can thus be described by a velocity-independent processing time $\left(R T_{0}=\right.$ $194 \mathrm{msec}$ ), a function for the delay for detectors of relative motion ( $\tau=80 / \sqrt{ } v \mathrm{msec}$ ), and a critical distance of $0.15 \mathrm{deg}$ for detection of displacement. 

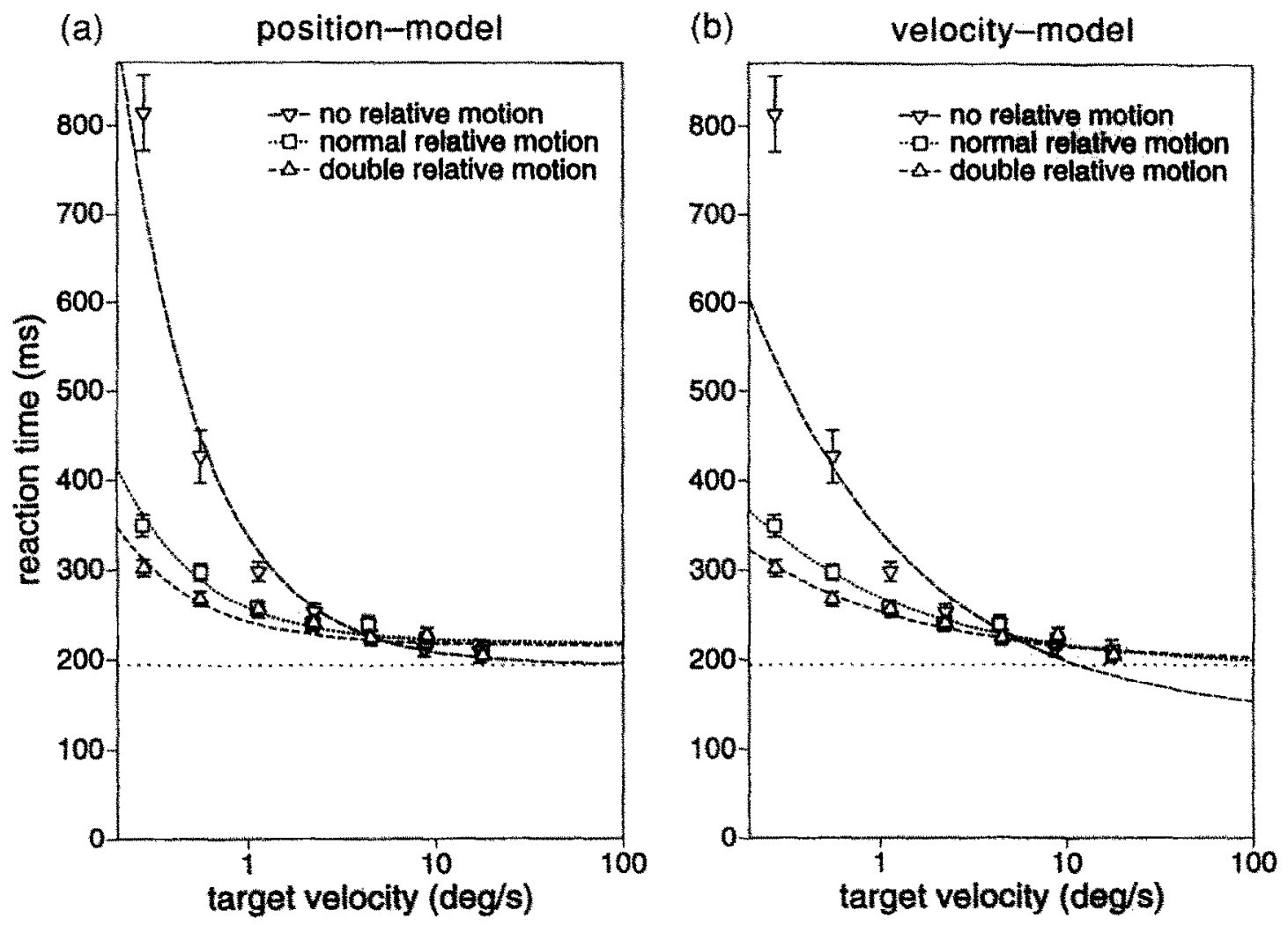

FIGURE 2. (a) Reaction time as a function of target velocity, for the three relative motion conditions. The curves are fits of the position model [equation (1)] to the data points. (b) The same data points as in (a); the curves are fits of the velocity model [equation (2)]. The dashed horizontal lines indicate the asymptotic value of $R T_{0}=194 \mathrm{msec}$. This is the average of the values for the best fits (either position model or velocity model) to the three relative motion conditions.

\section{DISCUSSION}

We can compare our results with those of several other studies. Tynan and Sekuler (1982) used a moving stimulus and a fixation point in their reaction-time experiment. Their subjects therefore always saw relative motion, so that we would expect the velocity model to fit their data better than the position model. This is indeed the case: as in our experiment with normal relative motion, the velocity model fits the data considerably better (smaller $\chi^{2}$ ) than the position model. From a study on the perception of motion in noisy random pixel arrays (relative to a fixation point), van Doorn and Koenderink (1982) reported a delay of motion detectors of: $\tau=89 v^{-0.4} \mathrm{msec}$. Although they used a totally different paradigm, this result is very similar to what we find in our study (see Fig. 3).

Gellman and Carl (1991) measured saccadic latency to a ramp displacement of a point target without visual reference. According to our hypothesis, their experiment tested the detection of (absolute) displacement. To model their results, they indeed used the model of equation (1). This yielded a value for $d$ of $0.14 \mathrm{deg}$, which is almost identical to the value we find. Fitting the velocity model to their data results in a considerably worse fit.

One can expect that the displacement detection threshold $d$ is related to the normal variability in the position at which we direct our eyes. Collewijn, Ferman and van den Berg (1988) reported that the standard deviation of horizontal eye position for subjects fixating a target on a random-dot background with the head fixed is 0.18 deg. Ferman, Collewijn, Jansen and van den Berg (1987) reported a standard deviation of the gaze of $0.11 \mathrm{deg}$ for subjects fixating a spot without a background while keeping their (free) head more or less stationary. These two experiments yield values for

TABLE 1. Parameters obtained by fiting the results of the experiments to the two models (see text)

\begin{tabular}{lccccccc}
\hline & \multicolumn{2}{c}{ Position model } & \multicolumn{3}{c}{ Velocity model } \\
Relative & $R T_{0}$ (msec) & $d(\mathrm{deg})$ & $\chi^{2}$ & $R T_{0}(\mathrm{msec})$ & $\mathrm{c}(\sqrt{\mathrm{deg}} / \mathrm{sec})$ & $\chi^{2}$ \\
\hline motion & 194 & 0.143 & 13.5 & 131 & 212 & $64.8^{*}$ \\
Nome & 219 & 0.039 & 8.1 & 192 & 79 & 3.6 \\
Double & 216 & 0.026 & $17,6^{*}$ & 197 & 56 & 5.0 \\
\hline
\end{tabular}

*The fit can be rejected $(P<0.01)$. 


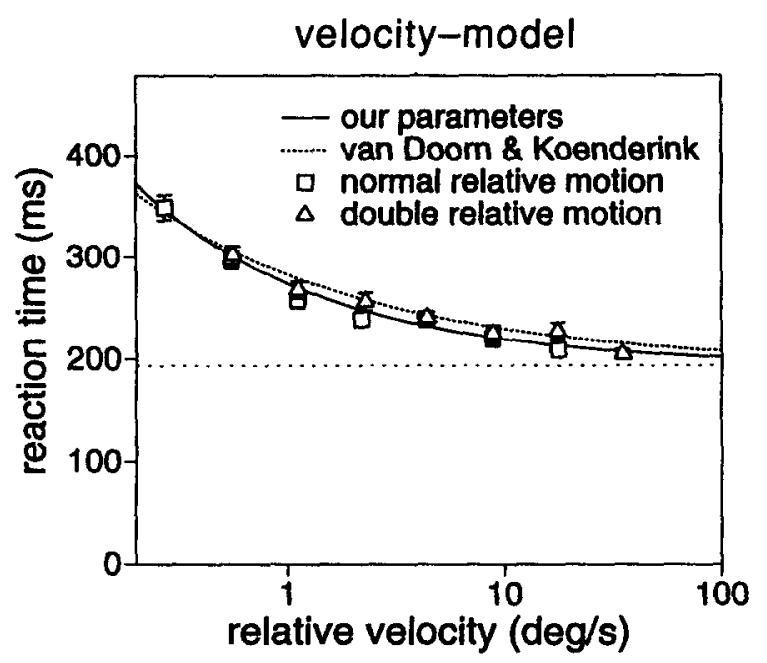

FIGURE 3. The responses in the two condiions with relative motion as a function of the velocity of the target relative to the background. Two models are plotted through the data: the velocity model of equation (2): $R T=194+80 / \sqrt{ } v$, and the model (and parameters) of van Doorn and Koenderink (1982): $R T=194+89 v^{-0.4} \mathrm{msec}$. (As van Doorn and Koenderink did not measure reaction times, and thus do not provide $R T_{0}$, we used our value for $R T_{0}$ for their model.)

the stability of gaze in the same range as our value for $d$.

The position model has one complicating factor. It assumes an (infinitely) long memory for position. Hansen and Skavenski (1977), however, showed that memory of eye position degrades within a few hundreds of milliseconds. This is probably an explanation for the (too) long reaction times for the slowest stimulus, and therefore for the systematic deviations between the values predicted by the position model and our measurements. As a consequence, the position model fits the data with relative motion better than the data without relative motion (see Table 1).

We can summarize our results (and those of other reaction-time experiments) as follows: if subjects are asked to react to motion onset, they will respond as soon as relative motion detectors are stimulated, or the target position changes to an extent that results in a larger retinal displacement than would arise from the natural variability in the direction of gaze.

\section{REFERENCES}

Ackroyd, M. H. (1973). Digital filters. London: Butterworths.

Anson, J. G. (1989). Effects of moment of inertia on simple reaction time. Journal of Motor Behavior, 2l, 60-71.

van den Berg, A. V. \& van de Grind, W. A. (1989). Reaction times to motion onset and motion detection thresholds reflect the properties of bilocal motion detectors. Vision Research, 29, 1261-1266.

Brenner, E. (1991). Judging object motion during a smooth pursuit eye movements: The role of optic flow. Vision Research, 31, 1893-1902.

Brenner, E. \& Smeets, J. B. J. (1993). Hitting a running spider on a moving background. Investigative Ophthalmology and Visual Science, 34, 706.

Collewijn, H. (1972). Latency and gain of the rabbit's optokinetic reactions to small movements. Brain Research, 36, 59-70.

Collewijn, H., Ferman, L. \& van den Berg, A. V. (1988). The behavior of human gaze in three dimensions. Annals of the New York Academy of Sciences, 545, 105-127.

van Doorn, A. J. \& Koenderink, J. J. (1982). Temporal properties of the visual detectability of moving spatial white noise. Experimental Brain Research, 45, 179-188.

Exner, S. (1888). Ueber optische Bewegungsempfindungen. Biologisches Centralblatt, 8, 437-448.

Ferman, L., Collewijn, H., Jansen, T. C. \& van den Berg, A. V. (1987). Human gaze stability in the horizontal, vertical and torsional direction during voluntary head movements, evaluated with a three-dimensional scleral induction coil technique. Vision Research, $27,811-828$.

Gellman, R. S. \& Carl, J. R. (1991). Motion processing for saccadic eye movements in humans. Experimental Brain Research, 84, 660-667.

Hansen, R. M. \& Skavenski, A. A. (1977). Accuracy of eye-position information for motor control. Vision Research, 17, 919-926.

Press, W. H., Flannery, B. P., Teukolsky, S. A. \& Vetterling, W. T. (1987). Numerical recipes in $C$, the art of scientific computing. Cambridge: Cambridge University Press.

Tynan, P. D. \& Sekuler, R. (1982). Motion processing in peripheral vision: Reaction time and perceived velocity. Vision Research, 22 , $61-68$. 